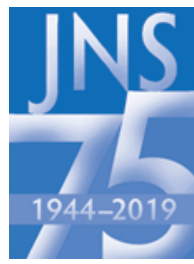

\title{
Recent advances in the neurosurgical treatment of pediatric epilepsy
}

\author{
JNSPG 75th Anniversary Invited Review Article
}

Jarod L. Roland, MD, and Matthew D. Smyth, MD

Department of Neurological Surgery, St. Louis Children's Hospital, Washington University in St. Louis, St. Louis, Missouri

\begin{abstract}
The field of epilepsy surgery has seen tremendous growth in recent years. Innovative new devices have driven much of this growth, but some has been driven by revisions of existing products. Devices have also helped to rejuvenate existing procedures, as in the case of robotic assistance for electrode placement for stereo-electroencephalography, and these devices have brought significant attention along with their introduction. Other devices, such as responsive neurostimulators or laser interstitial thermal therapy systems, have introduced novel treatment modalities and broadened the surgical indications. Collectively, these advances are rapidly changing much of the landscape in the world of pediatric neurosurgery for medically refractory epilepsy. The foundations for indications for neurosurgical intervention are well supported in strong research data, which has also been expanded in recent years. In this article, the authors review advances in the neurosurgical treatment of pediatric epilepsy, beginning with trials that have repeatedly demonstrated the value of neurosurgical procedures for medically refractory epilepsy and following with several recent advances that are largely focused on less-invasive intervention.
\end{abstract}

https://thejns.org/doi/abs/10.3171/2018.12.PEDS18350

KEYWORDS pediatric; epilepsy; epilepsy surgery; review

$\mathrm{D}$ ESPITE the development of several new antiepileptic drugs (AEDs) over the last 30 years, there has been little change in the overall effectiveness of the treatment of epilepsy with medication. ${ }^{11}$ However, there have been numerous advances in the neurosurgical treatment of epilepsy that have expanded treatment options for patients and reduced morbidity via less-invasive procedures. Herein we review some of the most recent advances and trends in the neurosurgical treatment of pediatric epilepsy.

\section{Expanding the Evidence in Support of Epilepsy Surgery}

Before reviewing technical advances that are actively changing pediatric epilepsy surgery, we will summarize some relevant recent literature. Several new reports have sought to replicate prior studies and strengthen the evergrowing evidence in the literature supporting the role of surgery for epilepsy.

The landmark 2001 study by Wiebe et al. is one of the very rare randomized controlled trials (RCTs) in neurosurgery. ${ }^{66}$ Trials that involve randomization to surgery versus medical management are difficult to perform due to the dramatically different levels of care and risks in the 2 arms. Often investigators struggle with factors that hinder the quality of such trials, such as poor enrollment, high rates of crossover, and poor compliance to study protocols. However, in countries where elective surgery is often scheduled on a potentially lengthy waiting list while medical therapy continues in the interim, a rather unique surgical trial design can be employed. This was the scenario used by Wiebe and colleagues in Canada to enroll 80 patients, 40 of whom were assigned to immediate surgical treatment while the other 40 were treated with AEDs for 1 year. The primary outcome of seizure freedom at 1 year was achieved in $58 \%$ of the surgical group versus $8 \%$ of the medical group $(p<0.001)$. Secondary outcomes of fewer seizures and quality of life also significantly favored the surgical group. The authors report no loss to follow-up and no crossover from the medical to the surgical group, but

ABBREVIATIONS AED = antiepileptic drug; ANT = anterior nucleus of the thalamus; BOLD = blood oxygen level dependent; CCEP = cortico-cortical evoked potential; DBS = deep brain stimulation; ECoG = electrocorticography; ERSET = Early Randomized Surgical Epilepsy Trial; FCD = focal cortical dysplasia; HH = hypothalamic hamartoma; $\mathrm{LITT}=$ laser interstitial thermal therapy; RCT = randomized controlled trial; r-fMRI = resting-state functional MRI; RNS = responsive neurostimulation; SEEG = stereoelectroencephalography; VNS = vagus nerve stimulation.

SUBMITTED December 10, 2018. ACCEPTED December 27, 2018.

INCLUDE WHEN CITING DOI: 10.3171/2018.12.PEDS18350. 
4 patients (10\%) in the surgical group ultimately did not undergo surgery. There was 1 sudden unexplained death in the medical group and no death in the surgical group. ${ }^{66}$

Wiebe and colleagues' trial was the first to provide level 1 evidence ${ }^{31}$ for surgery compared to medical management alone in the treatment of refractory epilepsy. The study represents a monumental effort and provided groundbreaking results that left little doubt as to the role of surgery for medically refractory epilepsy. However, despite its clear conclusions, a vast number of patients continue with poorly controlled seizures without obtaining evaluation at a comprehensive epilepsy center where surgical options can be considered..$^{23}$ A 2012 analysis of the Nationwide Inpatient Sample database by Englot et al. revealed that the use of surgery for refractory epilepsy did not significantly change during the period 1990-2008, which spanned the 2001 publication of the report by Wiebe et al. that provided level 1 evidence supporting its use. ${ }^{23}$

A subsequent RCT sought to address early surgical intervention in medically refractory epilepsy. ${ }^{22}$ The Early Randomized Surgical Epilepsy Trial (ERSET) was conducted at 16 centers in the United States and randomized 38 patients with mesial temporal epilepsy to a standardized anteromesial temporal resection $(\mathrm{n}=15)$ or continued medical management $(n=23)$. As is common with many surgical trials, the study did have a nontrivial number of crossovers, with 1 patient in the surgical arm never receiving surgery and 7 in the medical arm undergoing surgery prior to the 2-year follow-up point. Results revealed a greater rate of seizure freedom at 2 years' follow-up in the surgery group $(73 \%)$ than in the medically treated group $(0 \%)$. Quality-of-life metrics in the intention-to-treat analysis appeared higher in the surgical group (12.6 vs 4.0 in the medical group), but the difference did not reach statistical significance $(p=0.08)$. When the data from those who underwent surgery in the medical group were excluded, the difference was greater (12.8 surgical vs 2.8 medical) and reached statistical significance $(\mathrm{p}=0.01)$. Nevertheless, ERSET only included 2 patients younger than 17 years, limiting its direct applicability to pediatric neurosurgery.

Recently, a group in India replicated the Wiebe trial design in children. ${ }^{19}$ A total of 116 pediatric patients (age $\leq 18$ years) were randomized in a similar fashion to immediate neurosurgical treatment (57 patients) or continued medical therapy (59 patients). In contrast to the relatively uniform temporal lobe resections performed in ERSET ${ }^{22}$ and the Wiebe trial, ${ }^{66}$ the type of surgery performed in the Indian study varied based on each patient's individual needs. Procedures included resection (14 temporal, 12 extratemporal), disconnection (15 hemispherotomy, 10 corpus callosotomy), and hypothalamic hamartoma surgery (6). Dwivedi et al. report seizure freedom at the primary endpoint of 1 year in $77 \%$ of the surgery group compared to $7 \%$ of the medical group $(p<0.001) .{ }^{9}$ The collective results replicated across 3 RCTs provide the highest level of medical evidence for the role of surgery in medically refractory epilepsy.

Long-term retrospective studies are useful to ascertain sustained seizure response. The largest case series to date of temporal lobe epilepsy surgery in pediatric patients was recently presented by Ormond et al. (2018), who provide data on 183 children with at least 12 months of follow-up (mean 42 months) ${ }^{47}$ They report satisfactory seizure outcome, defined as Engel class I or II, in 85\% of their cohort. Along with their institutional experience, the authors provide a review of the literature on pediatric temporal lobe epilepsy surgery and identified 2089 cases, with a satisfactory outcome achieved in $79 \% .^{47}$ These outcomes in a retrospective cohort and large literature review are consistent with those of the RCTs described above. Together these studies provide strong and consistent data to support the role of neurosurgery for the treatment of medically refractory epilepsy in pediatric patients.

In a prospective cohort study published in 2013, Edelvik et al. ${ }^{21}$ report on pediatric patients at 5 and 10 years after they underwent surgery for epilepsy. The patients were identified through a Swedish national registry, and all had been 18 years old or younger at the time of surgery. Follow-up was performed via structured telephone at two time points: 5-year postoperative data were obtained in 42 cases (out of 48) and 10-year data in 46 (out of 60), for a combined long-term follow-up in 88 pediatric cases. Thirteen pediatric patients who underwent presurgical workup but did not proceed to surgery were similarly followed up at a mean of 8.8 years after the workup and served as a cross-sectional control group. In this combined group, $44 \%$ of the patients who were treated surgically achieved seizure freedom compared to none in the control group ( $\mathrm{p}$ $<0.0005)$. Of those who were seizure free at 10 years, $86 \%$ had stopped taking AEDs. The results of this prospective cohort study support the findings of the retrospective study described above and are also consistent with the results of the RCTs.

A large number of patients continue to suffer poorly controlled epilepsy without surgical evaluation despite several consistent and high-quality studies reporting significant numbers of pediatric patients achieving seizure freedom, seizure reduction, and increased quality of life. One nontrivial barrier for many patients, families, and referring practitioners is the morbidity associated with open craniotomy. This barrier persists despite published rates of complications from high-volume epilepsy centers that are quite low and the nontrivial morbidity and mortality of poorly controlled epilepsy. The morbidity associated with surgery is typically concentrated at the immediate perioperative period. In contrast, morbidity from poorly controlled epilepsy is diffuse and spread out over a lifetime. To address these concerns, many pediatric neurosurgeons have adapted to provide new and revitalized less-invasive treatment options.

\section{Endoscopic Disconnection}

As neurosurgeons become more familiar with tools of minimally invasive surgery, such as endoscopes, they have naturally adopted these tools for procedures used for epilepsy surgery. One example is the endoscopic hemispherotomy. The modern functional hemispherotomy can be performed through a variety of techniques, including the modified functional hemispherectomy, peri-insular hemispherotomy, and parasagittal hemispherotomy. ${ }^{1}$ With the addition of an endoscope, the hemispherotomy can be 
performed through a narrower window of approximately 4 $\times 3 \mathrm{~cm}$ or $4 \times 2 \mathrm{~cm} .{ }^{9,65}$

Chandra et al. subsequently added to the technique, as described in their 2018 article, by using a hybrid endoscopic setup that sits between the extracranial position of an exoscope and the near-to-field location of a narrow endoscope. ${ }^{10}$ They used a thicker $(10-\mathrm{mm})$ endoscope with a wider field of view to position the end of the scope further away from the working field but still intracranially, providing a less hindered space for movement. In addition, they compared several metrics of the open versus endoscopic hemispherotomies and reported less blood loss (mean 719 vs $210 \mathrm{ml}$, respectively, $\mathrm{p}=0.02)$, shorter hospital stay $(18.6$ vs 14.6 days, respectively, $\mathrm{p}=0.049$ ), and a nonsignificant difference in operative time (352 vs 302 minutes, respectively, $\mathrm{p}=0.06) .^{10}$

Part of the hemispherotomy entails a disconnection of the corpus callosum, and so the endoscopic techniques learned for one procedure directly translate to the other. ${ }^{59}$ In a 2017 article, Smyth et al. detail the procedures for open and endoscopic corpus callosotomy, both of which they perform through a frontal craniotomy, with the craniotomy in the endoscopic version being smaller. ${ }^{57}$ In a 2016 paper, Sood et al. also describe a posterior approach modification for endoscopic corpus callosotomy. They argue that the falx is much closer to the corpus callosum when approached from a posterior trajectory, necessitating less dissection of the interhemispheric fissure to complete the procedure..$^{58}$

\section{Stereo-Electroencephalography}

Stereotactic placement of multiple intraparenchymal depth electrodes for extraoperative intracranial electrophysiology monitoring can be performed with relatively low risks and high yield through the stereo-electroencephalography (SEEG) technique. At many centers, SEEG is rapidly expanding in practice, often replacing surfacebased electrocorticography (ECoG) for many indications. Because SEEG utilizes a percutaneous implantation technique, the pain and recovery associated with traditional craniotomy is greatly reduced, but the risks of blind passage of a depth electrode must be carefully weighed in this trade-off.

SEEG was introduced over 50 years ago in Europe..$^{14,60}$ More recent advances in stereotactic technology, such as robot-assisted localization, and improved CT and MRI angiography, which allow lead placement to avoid vasculature without the need for invasive catheter angiography, ${ }^{34,42}$ have facilitated the widespread adoption of SEEG across many centers in the US. The improved precision and ease of placing multiple intracranial recording electrodes, along with benefits of avoiding craniotomy, have met the requirements for a minimally invasive yet highly effective diagnostic procedure.

Clinical case series of modern SEEG techniques have been relatively small in comparison to similar reports of open procedures. Nevertheless, several groups have begun reporting their experience. In a 2014 paper, GonzalezMartinez et al. describe their experience with 122 patients, including 21 in the pediatric age range. ${ }^{27}$ Overall, the 122 patients underwent placement of 1586 electrodes (mean 13 electrodes per patient), with a complication rate of $0.18 \%$ per electrode placed, or $2.5 \%$ of patients.

In a similar study, published in 2018, Goldstein et al. report on their experience, with a focus on pediatric patients. ${ }^{26}$ They report on 25 children who underwent 30 SEEG procedures involving implantation of a total of 342 electrodes. In their series, no major complications occurred, but they did observe 4 asymptomatic intracranial hemorrhages and 2 superficial scalp infections that were treated with oral antibiotics.

Investigation of insular epilepsy is a unique strength of SEEG. The precision afforded by advanced neuronavigation techniques allows for electrodes to be safely placed in the depths of the insula within the candelabra of middle cerebral artery branches. Owing to its deep-seated location between the opercula of the frontal and temporal lobes, the insula is not readily studied with surface ECoG electrodes. Although rare, seizures originating from the insula have been known since early reports in the 1940s and 1950s. s0,48 $^{30}$ Additionally, seizures originating from the insula may easily masquerade as lobar in origin, potentially leading to false localization of the seizure onset zone and subsequent poor epilepsy response to surgery. ${ }^{35}$ For these reasons, the ability to study the insula with SEEG is an important tool in an epilepsy surgeon's armamentarium. ${ }^{20}$

An exemplar case of using SEEG to study the insula is illustrated in Fig. 1. This patient previously underwent craniotomy at 7 years of age for monitoring with surface ECoG electrodes followed by resection. However, the patient had a suboptimal seizure response. At this point, an insular onset was suspected, so the patient returned for percutaneous placement of leads around the prior resection site, including the insula, using the SEEG technique and the ROSA (Medtech/Zimmer Biomet) robotic guidance arm (Fig. 1A). Several electrodes were placed through the prior craniotomy bone flap and others through adjacent skull (Fig. 1B and C). A total of 74 electrodes were placed (Fig. 1D), leading to a successful localization of the seizure onset zone.

An early experience reported by Desai et al. in 2011 involved SEEG implantation of a total of 29 insular electrodes in 20 patients. The Leksell frame and stereotactic arc (Elekta AB) were used with a SurgiScope workstation (Intelligent Surgical Instruments and Systems) for electrode placement. The authors observed no significant morbidity in their series, although they did note 1 nonsatisfactory placement. ${ }^{18}$ In a 2018 publication, Tomycz et al. report on a larger series of cases in which insular electrodes were placed, with a focus on pediatric patients. They similarly used a combination of the Leksell frame and arc (Elekta AB) for electrode placement but with the Stealth FrameLink neuronavigation system (Medtronic, Inc.). They placed 74 insular electrodes in 49 patients ranging in age from 3 to 20 years. The majority of insular electrode placements in this series (84\%) were performed during open craniotomy for simultaneous surface ECoG placement. The authors report no major complications in their case series, but they note that return to the operating room was required for revision of 1 insular electrode. In addition, the authors briefly describe an additional patient 

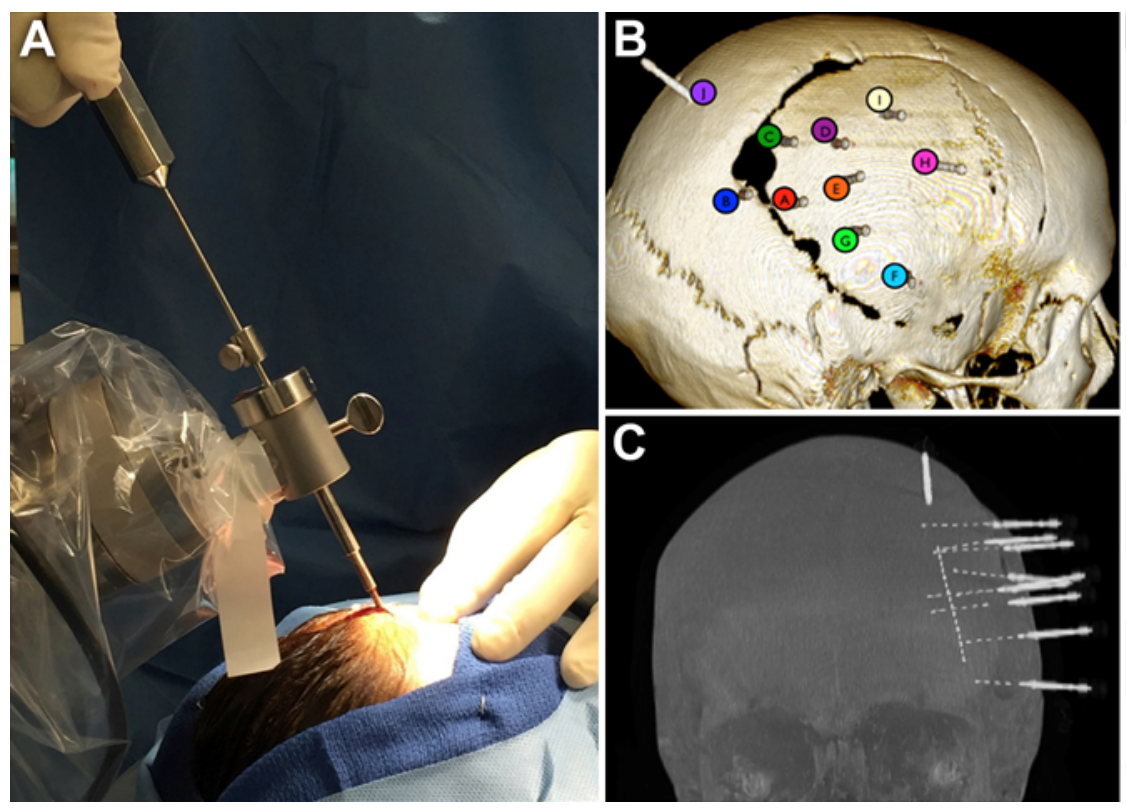

\begin{tabular}{|c|c|c|}
\hline \multirow{2}{*}{\multicolumn{3}{|c|}{$\begin{array}{l}\text { Entry Location } \\
\text { Posterior resection, } \\
\text { inferior }\end{array}$}} \\
\hline & & \\
\hline & $\begin{array}{l}\text { Posterior resection, } \\
\text { far posterior }\end{array}$ & 8 \\
\hline & $\begin{array}{l}\text { Posterior resection, } \\
\text { superior }\end{array}$ & 4 \\
\hline & $\begin{array}{l}\text { Posterior lesion, } \\
\text { superior }\end{array}$ & 8 \\
\hline & $\begin{array}{l}\text { Inferior-posterior } \\
\text { resection }\end{array}$ & 6 \\
\hline & $\begin{array}{l}\text { Superior temporal gyrus, } \\
\text { posterior }\end{array}$ & 4 \\
\hline & $\begin{array}{l}\text { Superior temporal gyrus, } \\
\text { anterior }\end{array}$ & 6 \\
\hline & $\begin{array}{l}\text { Anterior resection, } \\
\text { inferior }\end{array}$ & 8 \\
\hline & $\begin{array}{l}\text { Anterior resection, } \\
\text { superior }\end{array}$ & 8 \\
\hline & Posterior paramedian & 16 \\
\hline Total & & 74 \\
\hline
\end{tabular}

FIG. 1. SEEG placement for monitoring. The SEEG technique is illustrated, beginning with the placement of the anchoring bolt using the ROSA robotic guidance arm (A). This patient previously underwent a craniotomy for surface ECoG, and the current SEEG electrodes are placed percutaneously through the prior bone flap as well as adjacent skull (B). Electrodes are visualized by maximal intensity projection of a CT scan (C). A total of 74 electrodes were placed at targeted locations (D). Figure is available in color online only.

at their institution, outside of their case series, who suffered a fatal hemorrhage during removal of an SEEG lead followed by placement of a laser fiber within a structurally abnormal and atrophic insula. ${ }^{11}$ Such a scenario serves as a reminder of the catastrophic potential of operating near intracranial arteries. Nevertheless, these case series provide strong support for the safe placement of SEEG electrodes in the insula and for effective monitoring of insula-onset epilepsy, which is often difficult to diagnose.

\section{Laser Interstitial Thermal Therapy}

The minimally invasive diagnostic approach of SEEG is nicely complemented by laser interstitial thermal therapy (LITT) for therapeutic intervention. The surgical procedure for LITT has a similar minimally invasive profile. ${ }^{12}$ The technical aspects of placing SEEG electrodes and LITT laser fibers are similar, often using the same navigation frame and/or robotic assistance for both procedures. LITT is made possible by an advanced MRI sequence that allows for real-time thermography. More specifically, MRI thermography is able to detect changes in temperature from a set reference while heating is achieved with a laser power source. It can be performed either in an intraoperative MRI surgical suite or in a standard diagnostic MRI unit upon transfer from the operating room, where the laser fiber is first placed.

Two MRI-guided LITT systems are currently widely available. The NeuroBlate (Monteris Medical) system uses a carbon dioxide-cooled laser system and has the option for a more directional side-firing laser as well as a diffusion tip that heats in an omnidirectional pattern. The NeuroBlate system allows for manipulation of the rota- tion (with the side-firing configuration) and depth of the laser remotely via robotic control from the command station. The Visualase (Medtronic, Inc.) system uses a salinecooled laser probe and is available with a smaller-diameter probe. The smaller-diameter probe can be particularly advantageous for long thin structures, such as the corpus callosum. Adjustments to the depth of the laser are performed manually while the patient is in the MRI unit.

\section{LITT for Lesions}

LITT is tremendously useful for deep-seated lesions that may be difficult to treat safely with open surgery and provides an alternative to radiosurgery, avoiding the risks of ionizing radiation in children. Lesions that are relatively small (typically less than $2.5 \mathrm{~cm}$ in diameter) or cylindrical are particularly well suited for LITT due to the greater ease of conforming a zone of ablation to the full lesion with such geometry. This feature makes LITT an attractive option for treating a variety of small focal lesions that cause epilepsy, such as focal cortical dysplasia (FCD), tuberous sclerosis complex, and periventricular tumors such as subependymal giant cell astrocytoma (SEGA) ${ }^{6,16,17,41,62}$

The hypothalamic hamartoma $(\mathrm{HH})$ is one such stereotypical deep-seated epileptogenic lesion. Given the deepseated location and the morbidity of an open procedure, some institutions have begun using LITT as first-line therapy for HH. ${ }^{6}$ In a 2013 publication, Wilfong and Curry report a case series of 14 HHs treated with LITT; 13 of the 14 patients were pediatric and 1 was 20 years of age. The authors describe ultimately achieving seizure freedom in 9 of 10 patients who had at least 6 months of follow-up, including 1 patient who required 2 treatments. In their complete series, the only noted complication was an asymp- 

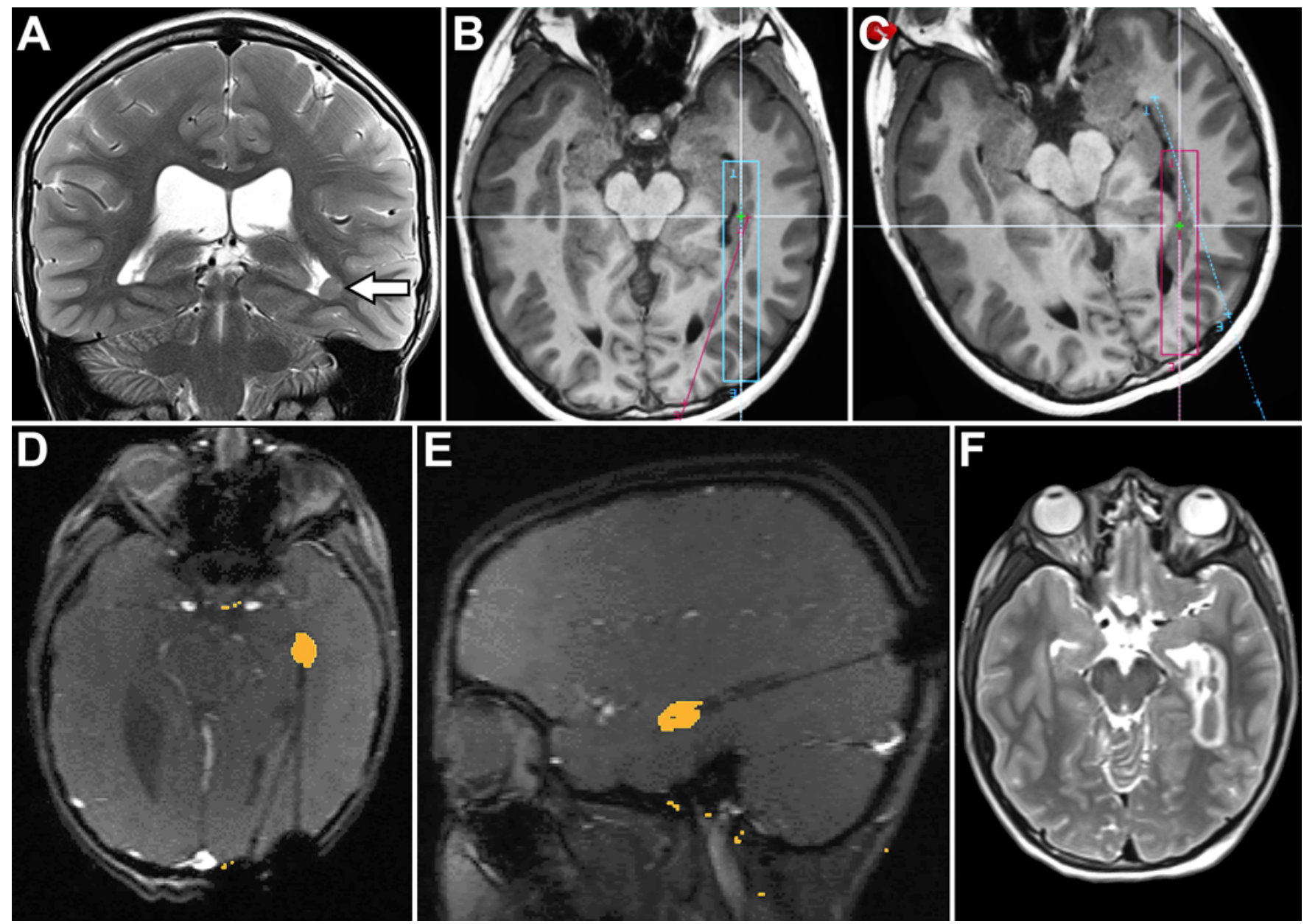

FIG. 2. LITT treatment of periventricular heterotopia. A periventricular nodular heterotopia is seen in the lateral wall of the temporal horn on coronal T2-weighted MRI (A). Two laser fibers are used to target the heterotopia along its anterior (B) and posterior (C) portions visualized on axial T1-weighted MRI. Each laser fiber used in successive treatments (D and E) to achieve ablation of the entirety of the heterotopia, where orange blocking designates area of treatment on oblique axial (D) and sagittal (E) MRI thermometry images. Treatment effect is confirmed on postoperative axial T2-weighted imaging (F). The images are presented in standard radiologic orientation. Figure is available in color online only.

tomatic subarachnoid hemorrhage that was seen on routine postoperative MRI. ${ }^{67}$ In a 2016 paper, Rolston and Chang describe their technique for treating $\mathrm{HH}$ using the same LITT system as Wilfong and Curry. The 2 patients Rolston and Chang report on were seizure free without complication at 5 and 7 months' follow-up. ${ }^{51}$ In another 2016 paper, Buckley et al. report on a series of 12 mixed lesional cases of refractory epilepsy treated by LITT. Six of the patients had HHs and had Engel class I or II outcomes. ${ }^{7}$ In a 2018 publication, $\mathrm{Xu}$ et al. describe their institutional series of cases involving adult and pediatric patients undergoing LITT for $\mathrm{HH}$. They report $80 \%$ seizure freedom in 15 of their patients who had gelastic seizures. They note that $39 \%$ of the patients in their series had immediate complications and $22 \%$ had persistent complications. ${ }^{68}$

While HHs are frequently small circular lesions, mesial temporal sclerosis can be viewed as a cylindrical lesion extending along the length of the mesial temporal lobe. Such a conformation is also readily amenable to LITT; the laser fiber is first placed at the depth of the temporal lobe target, then progressively backed up as successive sites are ablated..$^{29}$ In this fashion, concentric rings are effectively stacked to form a cylindrical zone of tissue ablation. This approach from an occipital entry and cannulation in the posterior-anterior direction along the long axis of the amygdalohippoampal complex is the most commonly reported. ${ }^{46}$ Data on pediatric cases have been reported more often than data on adult cases. In their recent (2017) review of 10 studies of LITT for mesial temporal lobe epilepsy, North et al. report Engel class I outcome in $63 \%$ of 78 cases (64\% of the 47 with at least 1 year of follow-up). ${ }^{46}$

LITT ablation of a nodular heterotopia in a pediatric patient is demonstrated in Fig. 2. This patient, who was 13 years old at the time of treatment, had medically refractory epilepsy that localized on surface EEG to the left temporal lobe. MRI revealed a nodular heterotopia along the left lateral wall of the temporal horn of the lateral ventricle (Fig. 2A). Two laser trajectories were required to ablate along the entire length of the heterotopia, from its anterior-most extent (Fig. 2B) to the posterior extent as it curved medi- 
ally (Fig. 2C). Each laser fiber was used to ablate along its length to create a cylindrical treatment zone (Fig. 2D and E), which was confirmed on postoperative imaging (Fig. 2 F). The patient was able to be discharged home on postoperative day 1 and has been seizure free ever since.

\section{LITT for Callosotomy}

In a video article published in 2018, Karsy et al. detail the procedure for performing an anterior two-thirds callosotomy with LITT. They used a Leksell head frame and stereotactic arc for navigation to place 3 laser fibers with the Visualase system. They noted a significant reduction in seizure frequency at 9 months' follow-up. ${ }^{37}$ In a 2017 paper, Singh et al. describe a theoretical approach to an anterior two-thirds callosotomy via a single trajectory and complete callosotomy via 2 trajectories, one from anterior and one from posterior. However, their proposed models have not been demonstrated in vivo. ${ }^{55}$

Patients with partial agenesis of the corpus callosum or return to surgery after partial callosotomy may represent ideal candidates for LITT callosotomy, as the smaller remnant corpus callosum is more easily targeted in its entirety. In a 2016 publication, Ho et al. report one such case of LITT for completion of a prior partial callosotomy. They were able to target the residual splenium of the corpus callosum with a single fiber. Four months postoperatively the patient had complete resolution of atonic seizures and an overall reduction in seizures by more than $50 \%{ }^{33}$

In a 2018 study, Lehner et al. report on 5 adult patients who underwent LITT for anterior corpus callosotomy ${ }^{40} \mathrm{Ef}-$ fective disconnection with LITT was validated by evaluating diffusion tensor imaging (DTI), resting-state functional MRI (r-fMRI), and cortico-cortical evoked potentials (CCEPs) in select patients undergoing concomitant SEEG. DTI revealed decreased fractional anisotropy in the region of the ablated corpus callosum relative to the remainder of the nonablated corpus callosum. Resting-state fMRI was used to confirm a decrease in interhemispheric functional connectivity with preservation of intrahemispheric functional connectivity, a technique that has previously been employed to demonstrate the role of the corpus callosum in interhemispheric functional connectivity ${ }^{36,49,50}$ Functional connectivity was also evaluated with resting ECoG signal analysis that similarly showed decreased interhemispheric connectivity between frontal lobes after anterior callosotomy. Collectively, these data provide strong support in a small number of adult patients studied before and after LITT callosotomy. ${ }^{40}$

\section{Vagus Nerve Stimulation}

The FDA first approved vagus nerve stimulation (VNS) in 1997 for adults and adolescents older than 12 years. Since that time, VNS has been frequently used off-label and in several studies in younger children. Blount et al. (2006) provided the first report of VNS for very young patients in a retrospective review of 6 patients age 5 years and less. ${ }^{3}$ In this cohort, $83 \%$ had a significant decrease in seizure frequency with no significant morbidity. The first RCT to examine VNS exclusively in pediatric patients was reported in 2012. ${ }^{38}$ In this pediatric trial, which was similar to those previously performed in adults, 41 patients (age
3-17 years) underwent high or low stimulation. Although no significant difference was seen between the 2 stimulation settings, the results at completion of an extended highstimulation period showed a $50 \%$ reduction in seizure frequency in $26 \%$ of the cohort. ${ }^{38}$ These and several other studies led to FDA approval in 2017 for VNS in patients age 4 years and greater with partial-onset seizures refractory to medical management. ${ }^{64}$

The primary VNS devices on the market are the VNS Therapy line from Cyberonics (a division of LivaNova, Inc.). The most recent models at the time of writing are the model 106 (AspireSR) and model 1000 (SenTiva). The model 106 introduced the option to use a cardiac sensing algorithm to detect rapid heart rate changes, which can be associated with seizure onset. Standard VNS is performed in an open-loop system with intermittent stimulation that is always on. With the newer models in the AutoStim cardiac sensing mode, an additional automated stimulation pulse can be provided by the device, similar to the manual magnet swipe previously performed by the patient or caregiver. This type of closed-loop stimulation can be useful for individuals who are physically or cognitively unable to perform a timely manual magnet swipe at seizure onset.

Two studies have reported that the AutoStim feature is well tolerated by patients. ${ }^{5,24}$ However, at least 1 complication has been reported in the literature. In a 2018 paper, Conti et al. describe short bouts of asystole and syncopal episodes after replacement of a model 103 with the model 1000. They report that the events resolved with explant of the 1000 and replacement with a new model $103 .{ }^{13}$

In addition to cardiac sensing, the model 1000 allows for programming a schedule for automatic advancement of stimulation parameters to improve ease of titrating stimulation after implantation. Automatic titration may be beneficial for those patients where repeat clinical visits are nontrivial. These added features, as well as a smaller-profile pulse generator, make for better efficacy and tolerance for patients undergoing VNS treatment.

\section{Responsive Neurostimulation}

Responsive neurostimulation (RNS) is an alternative, more regional, neuromodulation technique that functions in a closed-loop configuration. ${ }^{44}$ The NeuroPace RNS System (NeuroPace, Inc.) was approved by the FDA in 2013 for treatment in adults (age $\geq 18$ years) with refractory epilepsy and has been used off-label in pediatric patients. In 2014, Heck et al. reported the results of a multicenter double-blind RCT of RNS in 191 adult patients. After 2 months of recovery, a 3-month double-blinded period of stimulation versus sham showed a reduction in seizure frequency of $38 \%$ in the group receiving stimulation and $17 \%$ in the group receiving sham treatment. Then all participants continued with active stimulation, which led to a mean $44 \%$ and $53 \%$ seizure reduction at 1-year and 2-year follow-up, respectively. ${ }^{32}$ The latest long-term follow-up results were reported in a cohort of 256 adult patients who were followed for 9 years. The median seizure reduction was $75 \%$ in 168 patients still followed at 9 years. Throughout follow-up, $30 \%$ of patients achieved a 6 -month period of seizure freedom and $19 \%$ achieved 12 months. ${ }^{45}$ 

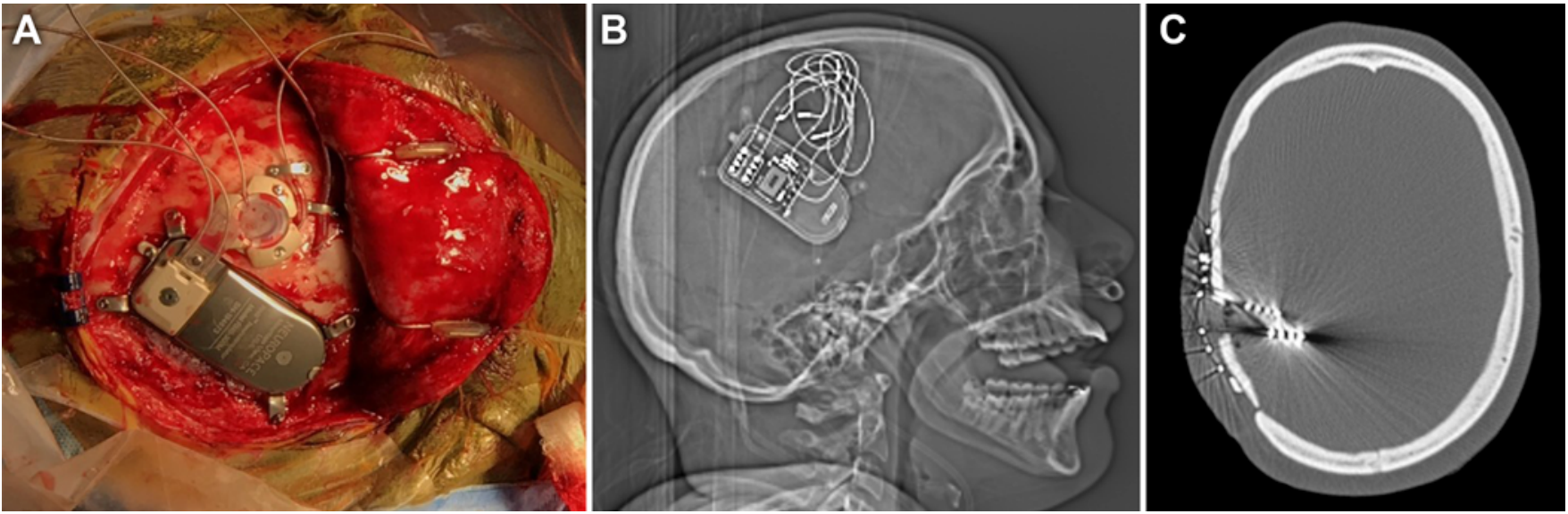

FIG. 3. RNS neuromodulation for seizure onset in eloquent cortex. A: Intraoperative photograph demonstrating the RNS system and implanted electrodes fixed to the skull. B: Postoperative lateral radiograph showing the device in place. C: CT image demonstrating the depth electrodes used for sensing and stimulation. Figure is available in color online only.

To date there have been very few case reports of pediatric patients undergoing RNS therapy for refractory epilepsy. In a 2018 case report, Singhal et al. describe their experience with RNS in a 16-year-old patient who underwent off-label RNS for a seizure focus that localized to eloquent speech cortex. ${ }^{56}$ Focal epilepsy localized to an eloquent area of cortex, which limits the available options for resection, is a scenario where the advantages of neuromodulation with RNS may be optimal. In Singhal and colleagues' patient, structural MRI revealed a transmantle sign suggestive of FCD in the left temporal lobe. Magnetoencephalography and positron emission tomography revealed concordant findings that localized to the same area. Invasive EEG monitoring with ECoG surface and depth electrodes subsequently confirmed the seizure onset zone in the area of the FCD with rapid spread to the overlying posterior superior temporal gyrus and angular gyrus. Unfortunately, bedside clinical mapping found disruption of speech with stimulation to part of the superior temporal gyrus, prohibiting a strategy of complete resection. Therefore, they opted for resection of the accessible FCD not associated with speech arrest accompanied by RNS placement during the same procedure. Interrogation of the ECoG recordings collected by the RNS device identified several electrographic seizures that corresponded with the patient's seizure diary, suggesting that partial resection was not curative. At initial follow-up the patient's daily seizures continued, albeit with reduced intensity and duration. Continued improvement was seen at 6 months when she was only experiencing auras several times per week without progression to alteration of awareness..$^{56}$

In the case presented in Fig. 1 (discussed previously in this article), SEEG localized the seizure onset zone to eloquent cortex. Therefore, the NeuroPace RNS system was implanted for neuromodulation. Figure 3A shows an intraoperative view of the device sitting in a window of bone that has been cut to its dimensions using a template. Next to the device are anchors for holding intracranial electrodes in place. The implant can be seen in a profile view (Fig. $3 \mathrm{~B})$ and in cross-section demonstrating the location of the depth electrodes (Fig. 3C). Despite the proximity to elo- quent cortex, no postoperative deficits were noted and the patient achieved a significant reduction in seizure frequency over the course of 2 years.

RNS is designed to deliver electrical stimulation in response to the onset of a seizure detected by digital signal analysis of the electrocorticogram. In this fashion the direct effect is primarily to abate progression. This effect may be responsible for the decrease in seizure duration and intensity reported at initial follow-up. Interestingly, early reports suggest there may also be a neuromodulatory effect on the seizure network over time that may also lead to decrease in seizure occurrence, separate from the effect of stopping a seizure once it is detected. Such an effect of stimulation leading to decreased seizure generation is similar to what has been reported with adult RNS ${ }^{43}$ and other neuromodulation devices such as VNS and DBS ${ }^{52}$ for epilepsy.

A progressive benefit of neuromodulation was also observed by Kokoszka et al. (2018), who describe 2 pediatric patients undergoing RNS. ${ }^{39}$ Their patients were a 14-yearold boy and a 9-year-old girl, both of whom achieved a beneficial response to RNS with a significant reduction in seizure frequency. In the 14-year-old patient, after an extensive prior surgical and invasive monitoring history, it was decided to place cortical surface electrodes over the left frontal and right temporal cortex as well as depth electrodes in the anterior nucleus of the thalamus. Over the first 3 months of stimulation there was not only a decrease in seizure duration, suggesting successful arrest of seizures once detected by the RNS, but also a decrease in the daily rate of detected abnormalities in the electrocorticogram. With the goal of additional seizure control, the team decided to reconfigure the device to use one of the thalamic electrodes for stimulation while using the temporal surface electrode for both seizure detection and stimulation. They report a $50 \%$ reduction in seizure frequency with this configuration. The second patient ( 9 years old) had a seizure onset zone that co-localized with eloquent speech and motor cortex on stimulation mapping in the left frontal and parietal lobes. Surface electrodes were placed over the putative seizure onset zone; 17 months later her seizure frequency had been reduced from 12 to 2 seizures per month, 
and at 21 months postoperatively she had been seizure free for 4 months. ${ }^{39}$

As in the above pediatric RNS case study, implantation of additional intracranial electrodes that can be interchanged in the RNS device provides an option for alternative recording and/or stimulation sites. Performing such an exchange can be done in a less-invasive procedure, as the proximal leads reside adjacent to the device in the extracranial space for easier access. Therefore, multiple stimulation and recording combinations can be configured in addition to varied stimulation parameters to optimize the monitoring and stimulation profiles for the individual patient.

Another unique function of the RNS device is its ability to record chronic ECoG for future analysis. Chan et al. (2018) describe the novel use of RNS devices that were implanted in 2 patients who had previously undergone invasive EEG monitoring for 3 weeks without successful identification of a seizure onset zone. They instead inserted depth electrodes in the hippocampus and in the neocortex overlying a periventricular nodular heterotopia identified on MRI in one patient, and in the hippocampus and orbitofrontal cortex of the second. In both patients these were sites where interictal spiking had been observed on invasive monitoring. Through correlation of chronic ECoG recordings via the RNS device with seizure diaries kept by the patients, they were able to detect seizures in the neocortex that rapidly spread to the hippocampus of the first patient and in the hippocampus, but not the orbitofrontal cortex, of the second. Both patients continued with neurostimulation, and at more than 1 year of follow-up, one patient had a $30 \%$ decrease in seizure frequency and the other a 50\% decrease. ${ }^{8}$ Given the challenges of invasive monitoring, particularly in very young pediatric patients, similar chronic ECoG recording strategies via the RNS device may prove to be valuable in the future.

\section{Deep Brain Stimulation}

Deep brain stimulation (DBS), a third type of neuromodulation, is well known to neurosurgeons for the treatment of movement disorders and has gained traction in recent years for treating medically refractory epilepsy (see the 2018 article by Zhou et al. ${ }^{70}$ for a recent review). DBS is typically performed in an open-loop fashion, similar to VNS. The results of a randomized double-blind trial involving stimulation of the anterior nuclei of the thalamus for epilepsy (SANTE) were published in 2010. ${ }^{25}$ SANTE included 110 adult patients (age $>18$ years, mean 36.1 years). The study included a 3-month blinded stimulation versus sham period, similar to that of previously mentioned neuromodulation trials. The between-groups difference in seizure frequency reduction was greater with increased time of stimulation such that at the end of the 3-month blinded period the stimulation group achieved a $40.1 \%$ reduction compared to a $14.5 \%$ reduction in the sham group. At the 2-year follow-up point, $54 \%$ of patients had achieved a $50 \%$ or greater reduction in seizure frequency $(56 \%$ median reduction). ${ }^{25}$ A subsequent long-term follow-up study by this group found continued improvement, with $68 \%$ of patients achieving 50\% reduction in seizure frequency at 5 years (69\% median reduction) as well as showing significant im- provement in quality-of-life metrics.$^{52}$ Further follow-up at 7 years reported long-term monitoring for neuropsychiatric adverse events that did not find objective evidence of cognitive decline or worsening depression, despite subjective complaints of memory problems and depression. ${ }^{63}$

In addition to the anterior nucleus of the thalamus (ANT), several other targets have been used for DBS in epilepsy. Alternative targets include the centromedian nucleus, subthalamic nucleus, ventral intermediate nucleus of the thalamus, nucleus accumbens, caudal zona incerta, mammillothalamic tract, fornix, posterior hypothalamus, and hippocampus. ${ }^{70}$ Only the ANT and hippocampus are supported by level 1 evidence thus far. ${ }^{15,25}$

The encouraging results of trials and cases series in adults has led to similar case reports and series in pediatric patients. Yan et al. performed a systematic review of the literature on DBS for epilepsy in children. In this study, which was published in 2018, they identified 21 studies describing a total of 40 cases in which pediatric patients (age range 4-18 years) underwent DBS for epilepsy. The various studies used a variety of targets, with the most frequent being the centromedian nucleus of the thalamus (used in 18 of 40 cases). Overall, a reduction in seizure frequency was reported in association with DBS in $85 \%$ of cases. ${ }^{69}$ Interestingly, 1 case study described the use of RNS with a deep stimulating electrode placed in the ANT in combination with neocortical surface electrodes on the temporal lobe. With this configuration, they reported improved seizure response using the combination of ANT stimulation and cortical sensing and stimulation. In this manner they effectively performed DBS in a closed-loop fashion. ${ }^{39}$

\section{Resting-State Functional MRI}

In addition to expanding the minimally invasive surgical procedures, recent work has sought to advance noninvasive diagnostic tools with advanced functional imaging. Resting-state functional MRI (r-fMRI) is a functional imaging technique that detects the low-frequency fluctuations in the blood oxygen level-dependent (BOLD) signal from the brain at rest. One of the unique strengths of $\mathrm{r}-\mathrm{fMRI}$ is the ability to identify intrinsic brain networks through correlations in the slow fluctuations of the BOLD signal. Seizure networks in epilepsy are postulated to disrupt these fluctuations and intrinsic networks. Ongoing studies are aimed at detecting specific alterations of the r-fMRI signal that may allow for its use as a noninvasive adjunct for lateralization and/or localization of seizures.

One such study measured $\mathrm{r}$-fMRI in pediatric patients with temporal lobe epilepsy. Shah et al. examined the phase offset, or lag, in the BOLD signal between hemispheres. In a cohort of 26 patients with temporal lobe epilepsy, they found that early or late lag relative to the mean signal predicted right or left seizure lateralization, respectively..$^{53}$ In another cohort of 38 patients with extratemporal epilepsy, Shah and colleagues report that the hemisphere with the greatest number of voxels with a significant lag relative to the mean signal correlated with the side of seizure lateralization..$^{54}$

In another study, Boerwinkle et al. used r-fMRI collected from a large cohort of 350 pediatric patients with 
medically refractory epilepsy who had undergone intracranial EEG monitoring for seizure localization to identify putative signs of an epileptogenic zone in the resting-state BOLD data. The authors compared the putative r-fMRI epileptogenic zone to the seizure onset zone determined by electrocorticography and found a $90 \%$ agreement. ${ }^{4}$

The strength of using r-fMRI as an adjunct to seizure localization comes from its relative ease. Resting-state fMRI is readily available on most clinical scanners already in use in typical pediatric hospitals. It is a modality that is very familiar to neurosurgeons and can be integrated with neuronavigation software. Most importantly, by its nature, r-fMRI does not require patient participation and can be successfully obtained under sedation-2 factors that are critically important in treating children who are at an immature age or developmentally delayed.

\section{Conclusions}

Recent years have seen significant advances in the neurosurgical treatment of pediatric epilepsy. Several of these advances stem from trials of modalities such as VNS, RNS, and DBS that were performed in adult patients. Of these treatment modalities, only VNS has since received FDA approval for use in pediatric patients as of the time of writing. However, off-label and on-study use of RNS and DBS has shown promise in pediatric patients. In addition, procedures that are more common in pediatric patients, such as corpus callosotomy and hemispherotomy, are continuing to be refined with less morbidity and less-invasive techniques.

Less-invasive procedures such as SEEG and LITT have the advantage of making the neurosurgical treatment of epilepsy more accessible and agreeable to patients, their families, and referring physicians. Reducing the risk and discomfort associated with open neurosurgical treatment may lower the barrier for referral and acceptance of treatment for those patients with medically refractory epilepsy who have not been evaluated at a comprehensive epilepsy center. It is possible, even likely, that less-invasive treatments may result in somewhat lower rates of seizure reduction compared to similar open procedures. This is suggested by recent data on outcomes from LITT for amygdalohippocampectomy in adults compared to historical seizure freedom rates with open craniotomy. ${ }^{2,28}$ However, even in the setting of a slightly lower seizure control rate, the decreased risk and morbidity may ultimately tip the risk-benefit ratio in favor of the minimally invasive option. An alternative paradigm of multiple smaller, staged procedures may accomplish meaningful seizure control rates while avoiding or minimizing the number of open craniotomies. SEEG has the advantage of not only being less invasive than subdural grid-based ECoG, but also facilitating a more flexible pace for determining and executing the second, therapeutic stage. With ECoG, the patient has already undergone a craniotomy for diagnostic monitoring as a first stage, and a second-stage craniotomy for explant and therapeutic intervention is necessary within a single, intense hospitalization. The second stage must be done in a timely manner to limit risk of infection or injury during invasive monitoring. Because SEEG does not require a craniotomy for placement or removal, deciding on the best therapeutic procedure is less time sensitive, and all of the treatment options either remain equally available during the index admission or can be scheduled electively in subsequent weeks, after comprehensive evaluation of SEEG data by the epilepsy team and detailed consideration of options by the patient and family.

Success with this style of less-invasive and possibly staged procedures is very promising. We expect that the near future will provide further data on these topics as more institutions incorporate these newer techniques and conduct outcome studies. Most importantly, we hope that these advances will continue to broaden the options available to our pediatric patients who previously have not had an acceptable surgical treatment option and will lead to an overall improved quality of life for patients suffering from epilepsy.

\section{References}

1. Baumgartner JE, Blount JP, Blauwblomme T, Chandra PS: Technical descriptions of four hemispherectomy approaches: from the Pediatric Epilepsy Surgery Meeting at Gothenburg 2014. Epilepsia 58 (Suppl 1):46-55, 2017

2. Bezchlibnyk YB, Willie JT, Gross RE: A neurosurgeon's view: laser interstitial thermal therapy of mesial temporal lobe structures. Epilepsy Res 142:135-139, 2018

3. Blount JP, Tubbs RS, Kankirawatana P, Kiel S, Knowlton R, Grabb PA, et al: Vagus nerve stimulation in children less than 5 years old. Childs Nerv Syst 22:1167-1169, 2006

4. Boerwinkle VL, Mohanty D, Foldes ST, Guffey D, Minard $\mathrm{CG}$, Vedantam A, et al: Correlating resting-state functional magnetic resonance imaging connectivity by independent component analysis-based epileptogenic zones with intracranial electroencephalogram localized seizure onset zones and surgical outcomes in prospective pediatric intractable epilepsy study. Brain Connect 7:424-442, 2017

5. Boon P, Vonck K, van Rijckevorsel K, El Tahry R, Elger CE, Mullatti N, et al: A prospective, multicenter study of cardiacbased seizure detection to activate vagus nerve stimulation. Seizure 32:52-61, 2015

6. Buckley R, Estronza-Ojeda S, Ojemann JG: Laser ablation in pediatric epilepsy. Neurosurg Clin N Am 27:69-78, 2016

7. Buckley RT, Wang AC, Miller JW, Novotny EJ, Ojemann JG: Stereotactic laser ablation for hypothalamic and deep intraventricular lesions. Neurosurg Focus 41(4):E10, 2016

8. Chan AY, Knowlton RC, Chang EF, Rao VR: Seizure localization by chronic ambulatory electrocorticography. Clin Neurophysiol Pract 3:174-176, 2018

9. Chandra PS, Kurwale N, Garg A, Dwivedi R, Malviya SV, Tripathi M: Endoscopy-assisted interhemispheric transcallosal hemispherotomy: preliminary description of a novel technique. Neurosurgery 76:485-495, 2015

10. Chandra PS, Subianto H, Bajaj J, Girishan S, Doddamani R, Ramanujam B, et al: Endoscope-assisted (with robotic guidance and using a hybrid technique) interhemispheric transcallosal hemispherotomy: a comparative study with open hemispherotomy to evaluate efficacy, complications, and outcome. J Neurosurg Pediatr [epub ahead of print November 9, 2018. DOI: 10.3171/2018.8.PEDS18131]

11. Chen Z, Brodie MJ, Liew D, Kwan P: Treatment outcomes in patients with newly diagnosed epilepsy treated with established and new antiepileptic drugs: a 30-year longitudinal cohort study. JAMA Neurol 75:279-286, 2018

12. Cobourn K, Fayed I, Keating RF, Oluigbo CO: Early outcomes of stereoelectroencephalography followed by MRguided laser interstitial thermal therapy: a paradigm for min- 
imally invasive epilepsy surgery. Neurosurg Focus 45(3):E8, 2018

13. Conti K, Falowski SM: Routine replacement of a vagal nerve stimulator generator leading to asystole. Epilepsy Behav Case Rep 10:122-123, 2018

14. Cossu M, Cardinale F, Castana L, Nobili L, Sartori I, Lo Russo G: Stereo-EEG in children. Childs Nerv Syst 22:766778, 2006

15. Cukiert A, Cukiert CM, Burattini JA, Mariani PP, Bezerra DF: Seizure outcome after hippocampal deep brain stimulation in patients with refractory temporal lobe epilepsy: a prospective, controlled, randomized, double-blind study. Epilepsia 58:1728-1733, 2017

16. Curry DJ, Gowda A, McNichols RJ, Wilfong AA: MR-guided stereotactic laser ablation of epileptogenic foci in children. Epilepsy Behav 24:408-414, 2012

17. Dadey DYA, Kamath AA, Leuthardt EC, Smyth MD: Laser interstitial thermal therapy for subependymal giant cell astrocytoma: technical case report. Neurosurg Focus 41(4):E9, 2016

18. Desai A, Jobst BC, Thadani VM, Bujarski KA, Gilbert K, Darcey TM, et al: Stereotactic depth electrode investigation of the insula in the evaluation of medically intractable epilepsy. J Neurosurg 114:1176-1186, 2011

19. Dwivedi R, Ramanujam B, Chandra PS, Sapra S, Gulati S, Kalaivani M, et al: Surgery for drug-resistant epilepsy in children. N Engl J Med 377:1639-1647, 2017

20. Dylgjeri S, Taussig D, Chipaux M, Lebas A, Fohlen M, Bulteau C, et al: Insular and insulo-opercular epilepsy in childhood: an SEEG study. Seizure 23:300-308, 2014

21. Edelvik A, Rydenhag B, Olsson I, Flink R, Kumlien E, Källén $\mathrm{K}$, et al: Long-term outcomes of epilepsy surgery in Sweden: a national prospective and longitudinal study. Neurology 81:1244-1251, 2013

22. Engel J Jr, McDermott MP, Wiebe S, Langfitt JT, Stern JM, Dewar S, et al: Early surgical therapy for drug-resistant temporal lobe epilepsy: a randomized trial. JAMA 307:922-930, 2012

23. Englot DJ, Ouyang D, Garcia PA, Barbaro NM, Chang EF: Epilepsy surgery trends in the United States, 1990-2008. Neurology 78:1200-1206, 2012

24. Fisher RS, Afra P, Macken M, Minecan DN, Bagić A, Benbadis SR, et al: Automatic vagus nerve stimulation triggered by ictal tachycardia: clinical outcomes and device performance-the U.S. E-37 trial. Neuromodulation 19:188-195, 2016

25. Fisher R, Salanova V, Witt T, Worth R, Henry T, Gross R, et al: Electrical stimulation of the anterior nucleus of thalamus for treatment of refractory epilepsy. Epilepsia 51:899-908, 2010

26. Goldstein HE, Youngerman BE, Shao B, Akman CI, Mandel AM, McBrian DK, et al: Safety and efficacy of stereoelectroencephalography in pediatric focal epilepsy: a single-center experience. J Neurosurg Pediatr 22:444-452, 2018

27. Gonzalez-Martinez J, Mullin J, Vadera S, Bulacio J, Hughes $\mathrm{G}$, Jones S, et al: Stereotactic placement of depth electrodes in medically intractable epilepsy. J Neurosurg 120:639-644, 2014

28. Gross RE, Stern MA, Willie JT, Fasano RE, Saindane AM, Soares BP, et al: Stereotactic laser amygdalohippocampotomy for mesial temporal lobe epilepsy. Ann Neurol 83:575587,2018

29. Gross RE, Willie JT, Drane DL: The role of stereotactic laser amygdalohippocampotomy in mesial temporal lobe epilepsy. Neurosurg Clin N Am 27:37-50, 2016

30. Guillaume MM, Mazars G: [Five cases of operated insular epileptogenic zone.] Soc Française Neurol:766-769, 1949 (Fr)

31. Harris RP, Helfand M, Woolf SH, Lohr KN, Mulrow CD,
Teutsch SM, et al: Current methods of the US Preventive Services Task Force: a review of the process. Am J Prev Med 20 (3 Suppl):21-35, 2001

32. Heck CN, King-Stephens D, Massey AD, Nair DR, Jobst BC, Barkley GL, et al: Two-year seizure reduction in adults with medically intractable partial onset epilepsy treated with responsive neurostimulation: final results of the RNS System Pivotal trial. Epilepsia 55:432-441, 2014

33. Ho AL, Miller KJ, Cartmell S, Inoyama K, Fisher RS, Halpern $\mathrm{CH}$ : Stereotactic laser ablation of the splenium for intractable epilepsy. Epilepsy Behav Case Rep 5:23-26, 2016

34. Ho AL, Muftuoglu Y, Pendharkar AV, Sussman ES, Porter $\mathrm{BE}$, Halpern $\mathrm{CH}$, et al: Robot-guided pediatric stereoelectroencephalography: single-institution experience. J Neurosurg Pediatr 22:489-496, 2018

35. Isnard J, Guénot M, Sindou M, Mauguière F: Clinical manifestations of insular lobe seizures: a stereo-electroencephalographic study. Epilepsia 45:1079-1090, 2004

36. Johnston JM, Vaishnavi SN, Smyth MD, Zhang D, He BJ, Zempel JM, et al: Loss of resting interhemispheric functional connectivity after complete section of the corpus callosum. J Neurosci 28:6453-6458, 2008

37. Karsy M, Patel DM, Halvorson K, Mortimer V, Bollo RJ: Anterior two-thirds corpus callosotomy via stereotactic laser ablation. Neurosurg Focus 44 (VideoSuppl2):V2, 2018

38. Klinkenberg S, Aalbers MW, Vles JSH, Cornips EMJ, Rijkers K, Leenen L, et al: Vagus nerve stimulation in children with intractable epilepsy: a randomized controlled trial. Dev Med Child Neurol 54:855-861, 2012

39. Kokoszka MA, Panov F, La Vega-Talbott M, McGoldrick PE, Wolf SM, Ghatan S: Treatment of medically refractory seizures with responsive neurostimulation: 2 pediatric cases. J Neurosurg Pediatr 21:421-427, 2018

40. Lehner KR, Yeagle EM, Argyelan M, Klimaj Z, Du V, Megevand P, et al: Validation of corpus callosotomy after laser interstitial thermal therapy: a multimodal approach. J Neurosurg [epub ahead of print November 16, 2018. DOI: 10.3171/2018.4.JNS172588]

41. Lewis EC, Weil AG, Duchowny M, Bhatia S, Ragheb J, Miller I: MR-guided laser interstitial thermal therapy for pediatric drug-resistant lesional epilepsy. Epilepsia 56:1590-1598, 2015

42. Miller BA, Salehi A, Limbrick DD, Smyth MD: Applications of a robotic stereotactic arm for pediatric epilepsy and neurooncology surgery. J Neurosurg Pediatr 20:364-370, 2017

43. Morrell MJ: Responsive cortical stimulation for the treatment of medically intractable partial epilepsy. Neurology 77:1295-1304, 2011

44. Morrell MJ, Halpern C: Responsive direct brain stimulation for epilepsy. Neurosurg Clin N Am 27:111-121, 2016

45. Nair DR, Morrell MJ: Nine-year prospective safety and effectiveness outcomes from the long-term treatment trial of the RNS system, in American Epilepsy Society Annual Meeting, 2018 (Abstract 2.075) (https://www.aesnet.org/ meetings_events/annual_meeting_abstracts/view/502690) [Accessed January 4, 2019]

46. North RY, Raskin JS, Curry DJ: MRI-guided laser interstitial thermal therapy for epilepsy. Neurosurg Clin N Am 28:545-557, 2017

47. Ormond DR, Clusmann H, Sassen R, Hoppe C, Helmstaedter C, Schramm J, et al: Pediatric temporal lobe epilepsy surgery in Bonn and review of the literature. Neurosurgery [epub ahead of print], 2018

48. Penfield W, Faulk ME Jr: The insula; further observations on its function. Brain 78:445-470, 1955

49. Pizoli CE, Shah MN, Snyder AZ, Shimony JS, Limbrick DD, Raichle ME, et al: Resting-state activity in development and maintenance of normal brain function. Proc Natl Acad Sci U S A 108:11638-11643, 2011 
50. Roland JL, Snyder AZ, Hacker CD, Mitra A, Shimony JS, Limbrick DD, et al: On the role of the corpus callosum in interhemispheric functional connectivity in humans. Proc Natl Acad Sci 114:13278-13283, 2017

51. Rolston JD, Chang EF: Stereotactic laser ablation for hypothalamic hamartoma. Neurosurg Clin N Am 27:59-67, 2016

52. Salanova V, Witt T, Worth R, Henry TR, Gross RE, Nazzaro $\mathrm{JM}$, et al: Long-term efficacy and safety of thalamic stimulation for drug-resistant partial epilepsy. Neurology 84:10171025,2015

53. Shah MN, Mitra A, Goyal MS, Snyder AZ, Zhang J, Shimony JS, et al: Resting state signal latency predicts laterality in pediatric medically refractory temporal lobe epilepsy. Childs Nerv Syst 34:901-910, 2018

54. Shah MN, Nguyen RD, Pao LP, Zhu L, CreveCoeur TS, Mitra A, et al: Role of resting state MRI temporal latency in refractory pediatric extratemporal epilepsy lateralization. J Magn Reson Imaging, 2018

55. Singh H, Essayed WI, Deb S, Hoffman C, Schwartz TH: Minimally invasive robotic laser corpus callosotomy: a proof of concept. Cureus 9:e1021, 2017

56. Singhal NS, Numis AL, Lee MB, Chang EF, Sullivan JE, Auguste KI, et al: Responsive neurostimulation for treatment of pediatric drug-resistant epilepsy. Epilepsy Behav Case Rep 10:21-24, 2018

57. Smyth MD, Vellimana AK, Asano E, Sood S: Corpus callosotomy-open and endoscopic surgical techniques. Epilepsia 58 (Suppl 1):73-79, 2017

58. Sood S, Asano E, Altinok D, Luat A: Endoscopic posterior interhemispheric complete corpus callosotomy. J Neurosurg Pediatr 25:689-692, 2016

59. Sood S, Marupudi NI, Asano E, Haridas A, Ham SD: Endoscopic corpus callosotomy and hemispherotomy. J Neurosurg Pediatr 16:681-686, 2015

60. Talairach J, Bancaud J: Stereotaxic approach to epilepsy, in Krayenbühl H, Maspes PE, Sweet WH (eds): Progress in Neurological Surgery. Basel: Karger Publishers, 1973, Vol 5, pp 297-354

61. Tomycz LD, Hale AT, Haider AS, Clarke DF, Lee MR: Invasive insular sampling in pediatric epilepsy: a single-institution experience. Oper Neurosurg (Hagerstown) 15:310-317, 2018

62. Tovar-Spinoza Z, Ziechmann R, Zyck S: Single and staged laser interstitial thermal therapy ablation for cortical tubers causing refractory epilepsy in pediatric patients. Neurosurg Focus 45(3):E9, 2018

63. Tröster AI, Meador KJ, Irwin CP, Fisher RS: Memory and mood outcomes after anterior thalamic stimulation for refractory partial epilepsy. Seizure 45:133-141, 2017
64. US Food \& Drug Administration: PMA P970003/ S207. Summary of Safety and Effectiveness Data (SSED). (https://www.accessdata.fda.gov/cdrh_docs/pdf/ p970003s207b.pdf) [Accessed January 4, 2019]

65. Wagner K, Vaz-Guimaraes F, Camstra K, Lam S: Endoscope-assisted hemispherotomy: translation of technique from cadaveric anatomical feasibility study to clinical implementation. J Neurosurg Pediatr [epub ahead of print November 2, 2018. DOI: 10.3171/2018.8.PEDS18349]

66. Wiebe S, Blume WT, Girvin JP, Eliasziw M: A randomized, controlled trial of surgery for temporal-lobe epilepsy. $\mathbf{N}$ Engl J Med 345:311-318, 2001

67. Wilfong AA, Curry DJ: Hypothalamic hamartomas: optimal approach to clinical evaluation and diagnosis. Epilepsia 54 (Suppl 9):109-114, 2013

68. Xu DS, Chen T, Hlubek RJ, Bristol RE, Smith KA, Ponce FA, et al: Magnetic resonance imaging-guided laser interstitial thermal therapy for the treatment of hypothalamic hamartomas: a retrospective review. Neurosurgery 83:1183-1192, 2018

69. Yan H, Toyota E, Anderson M, Abel TJ, Donner E, Kalia SK, et al: A systematic review of deep brain stimulation for the treatment of drug-resistant epilepsy in childhood. J Neurosurg Pediatr [epub ahead of print November 30, 2018. DOI: 10.3171/2018.9.PEDS18417]

70. Zhou JJ, Chen T, Farber SH, Shetter AG, Ponce FA: Openloop deep brain stimulation for the treatment of epilepsy: a systematic review of clinical outcomes over the past decade (2008-present). Neurosurg Focus 45(2):E5, 2018 (Erratum in Neurosurg Focus 45(5):E13, 2018)

\section{Disclosures}

The authors report no conflict of interest concerning the materials or methods used in this study or the findings specified in this paper.

\section{Author Contributions}

Conception and design: Smyth. Drafting the article: both authors Critically revising the article: both authors. Reviewed submitted version of manuscript: both authors. Administrative/technical/ material support: Smyth.

\section{Correspondence}

Jarod L. Roland: St. Louis Children's Hospital, Washington University in St. Louis, MO. rolandj@wustl.edu. 